\title{
A Case of Suspected Tuberculous Pleurisy and Coronavirus Disease 2019 Comorbidity
}

\author{
Yoh Yamaguchi ${ }^{1}$, Masao Hashimoto ${ }^{1}$, Susumu Saito ${ }^{1}$, Tie Morita ${ }^{1}$, Akinari Tsukada ${ }^{1}$, \\ Yusaku Kusaba ${ }^{1}$, Takashi Katsuno ${ }^{1}$, Manabu Suzuki ${ }^{1}$, Jin Takasaki ${ }^{1}$, Shinyu Izumi ${ }^{1}$, \\ Akihiro Matsunaga ${ }^{2}$, Yukihito Ishizaka ${ }^{2}$, Masayuki Hojo ${ }^{1}$ and Haruhito Sugiyama ${ }^{1}$
}

\begin{abstract}
:
A 33-year-old woman with a fever, cough, and pharyngitis was admitted after left-sided pleural effusion was detected. The fever and upper respiratory symptoms were confirmed, and she was diagnosed with coronavirus disease (COVID-19) after showing a positive polymerase chain reaction (PCR) test. After thoracentesis, pleural fluid revealed elevated adenosine deaminase values and a positive QuantiFeron test; tuberculous pleurisy was thus suspected. Subsequent SARS-CoV-2 PCR and anti-SARS-CoV-2 Spike IgG tests were negative, suggesting that the initial PCR result had been erroneous. However, we were unable to confirm this. Data concerning COVID-19 diagnostics are insufficient at present. It is important to make comprehensive judgments regarding the diagnosis and treatment of patients as well as public health.
\end{abstract}

Key words: tuberculous pleurisy, COVID-19, SARS-CoV-2

(Intern Med Advance Publication)

(DOI: 10.2169/internalmedicine.6920-21)

\section{Introduction}

The novel coronavirus infection (COVID-19) is a respiratory tract infection caused by Severe Acute Respiratory Syndrome Coronavirus 2 (SARS-CoV-2), and more than 200 million people worldwide were reported to have been infected as of August 2021 (1). Commonly seen symptoms include a fever, respiratory symptoms (cough and sore throat), headache, and fatigue (2). While discussion is ongoing regarding appropriate specimens for making a diagnosis, the current method commonly used involves testing nasopharyngeal swabs or sputum samples for SARS-CoV-2 using PCR. During the current pandemic, patients without symptoms contraindicating COVID-19 and complaining of a fever and upper respiratory symptoms undergo PCR tests to detect COVID-19.

\section{Case Report}

A 33-year-old woman presented with no notable history of disease and a history of contact with symptomatic individuals. Seven days prior to hospitalization, she developed a $39{ }^{\circ} \mathrm{C}$ fever and noticed pain on the left side of her chest. She underwent a PCR test at a local testing location, which came back negative. The patient visited the hospital for an emergency outpatient examination after she developed a headache, cough, sore throat, and sustained high fever. As shown in Fig. 2, the patient was hospitalized for the examination and treatment of mild decreased oxygenation $\left(\mathrm{SpO}_{2}\right.$ at $94 \%$, room air) and left-sided pleural effusion, which was confirmed through chest X-ray and thoracic computed tomography (CT).

For a diagnosis, a thoracostomy tube was inserted into the left-sided pleural effusion, and a light-yellow, serous, lymphocyte-dominated, exudative pleural fluid was extracted. Several differential diagnoses were considered for

${ }^{1}$ Department of Respiratory Medicine, Hospital, National Center for Global Health and Medicine, Japan and ${ }^{2}$ Department of Intractable Diseases, Research Institute, National Center for Global Health and Medicine, Japan

Received: February 2, 2021; Accepted: October 25, 2021; Advance Publication by J-STAGE: December 28, 2021

Correspondence to Dr. Yoh Yamaguchi, yoyamaguchi@hosp.ncgm.go.jp 


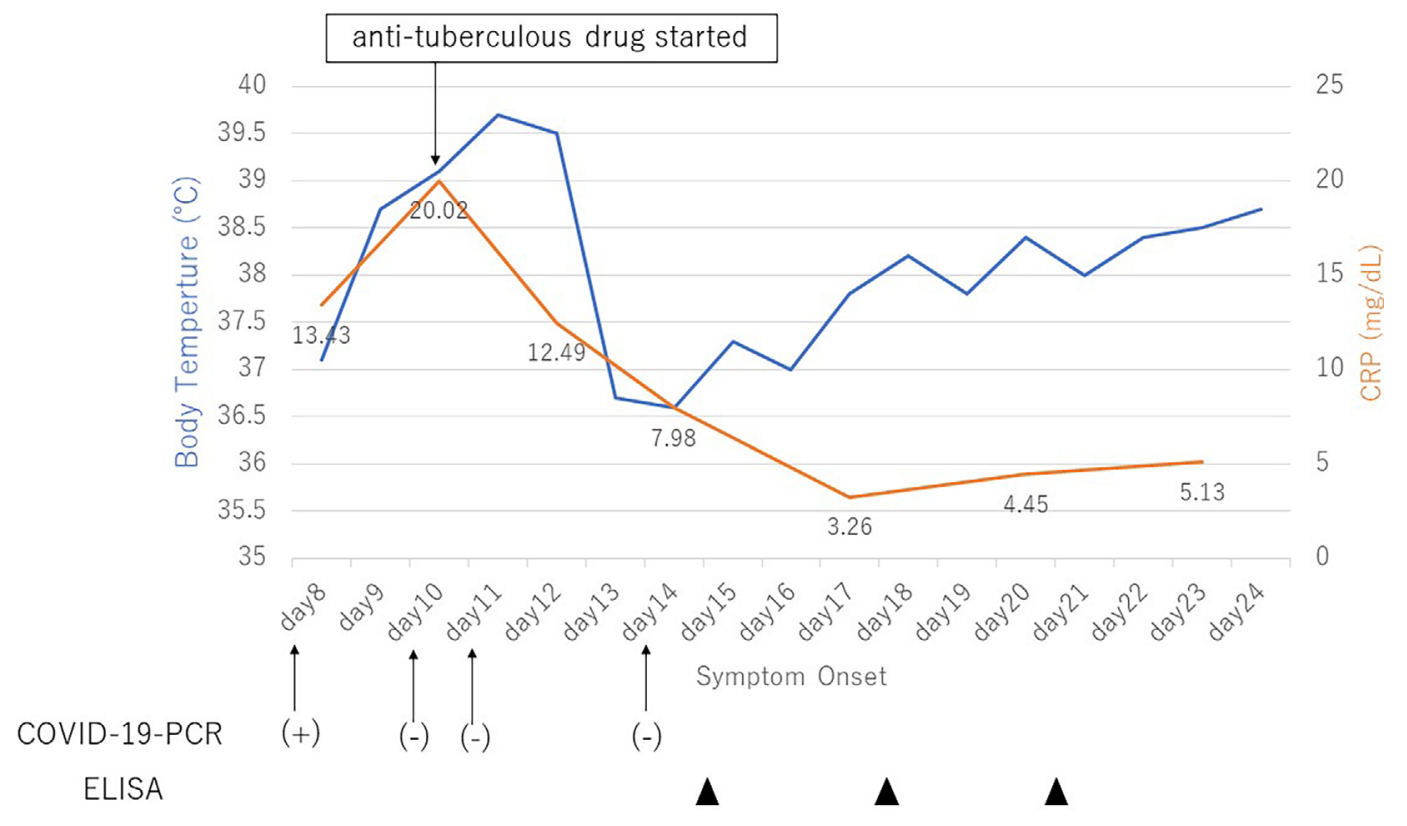

Figure 1. Clinical course of COVID-19-suspected patients.

the lateral pleural effusion, including tuberculous pleurisy, bacterial pleurisy, a connective tissue disease-related disorder, and a malignant tumor. In addition, given the COVID19 pandemic and the patient's fever and upper respiratory symptoms, COVID-19 was also considered as a potential diagnosis. A PCR test was performed, and a positive result for COVID-19 was obtained.

The patient had no ground-glass opacity in the lung field typically seen in patients with COVID-19. As COVID-19 does not typically result in lateral pleural effusion, the patient was first treated with general antibiotics (ceftriaxone on the day of hospitalization; ampicillin sulbactam on subsequent days) while awaiting the results of diagnostic tests for tuberculous pleurisy. However, over the course of several days, the patient's symptoms did not improve.

Three sputum smear tests for mycobacteria using ZiehlNeelsen staining were negative, and the sputum TB-PCR test was negative. However, a clinical diagnosis of tuberculous pleurisy was established owing to the high adenosine deaminase value $(105.2 \mathrm{U} / \mathrm{L})$ in the pleural effusion and positive QuantiFeron test. Upon consultation with the patient, treatment with anti-tuberculosis medication (HREZ: isoniazid [INH] $250 \mathrm{mg} /$ day + rifampicin [RFP] $600 \mathrm{mg} /$ day + ethambutol [EB] $750 \mathrm{mg} /$ day + pyrazinamide [PZA] 1,300 $\mathrm{mg} /$ day) was started, and a pleural biopsy was not performed for a bacteriological diagnosis. After starting the anti-tuberculosis treatment, blood tests showed improvement in the patient's inflammatory response and body temperature. The amount of pleural fluid drained by the thoracostomy tube was less than $100 \mathrm{~mL} /$ day, and the clinical course was favorable, so the tube was removed on the fifth day of hospitalization.

Thoracic CT was performed again to check for the presence of pulmonary tuberculosis. A portion of the lower-left lobe was atelectatic, making an evaluation difficult. How- ever, there were no findings suggesting active tuberculosis causing a high-volume of bacterial discharge. After more than two weeks of oral treatment with anti-tuberculosis medication, a mycobacterial smear test of sputum was negative. Therefore, the patient was deemed to have met the standards for hospital discharge with regard to tuberculosis infection.

While the PCR test for COVID-19 at the time of hospitalization was positive, it was suspected that this may have been a false positive; the CT scan lacked the typical groundglass opacity in the lung field, so a clinical diagnosis of tuberculous pleurisy was established. The patient's fever continued, but three nasal-swab PCR tests for COVID-19 were performed following hospitalization, and all had negative results. As the fever did not contradict tuberculous pleurisy, and more than two COVID-19-PCR tests were negative, the patient was discharged upon consultation with the presiding public health center.

A bacteriological diagnosis of active pulmonary tuberculosis and tuberculous pleurisy was confirmed 52 and 47 days post-hospitalization when Mycobacterium tuberculosis was cultured from the patient's sputum and pleural fluid, respectively. In addition, serum anti-SARS-CoV-2 Spike IgG titers, measured by an enzyme-linked immunosorbent assay (ELISA) (3) 8, 11 and 14 days (on days 15, 18 and 21, respectively) after the initial onset of symptoms, were not increased (Fig. 1, 2). Following discharge from the hospital, the patient continued treatment for tuberculous pleurisy with anti-tuberculosis medication.

\section{Discussion}

In this case study, we encountered a patient with upper respiratory tract symptoms, a fever, and unilateral pleural effusion. Because of the patient's symptoms and contact his- 


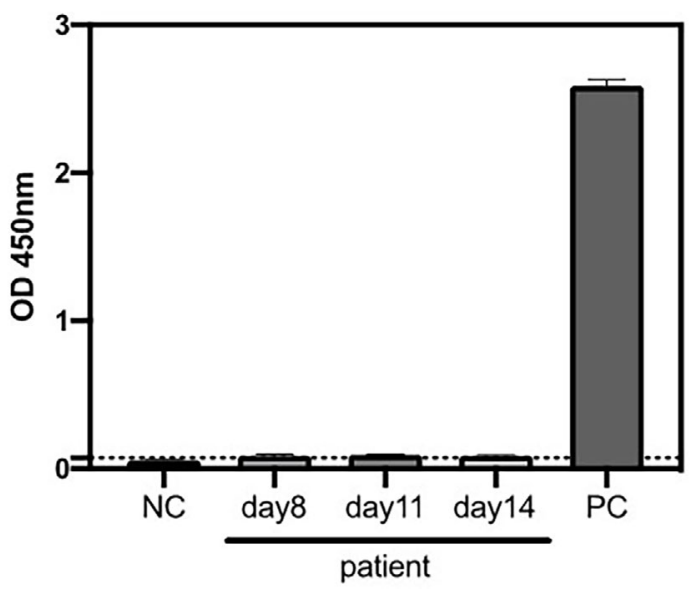

Figure 2. An ELISA of anti-SARS-CoV-2 Spike IgG in the patient serum samples. Serum samples of the patient at days 8 , 11 and 14 after hospitalization were analyzed by an anti-SARSCoV-2 Spike IgG ELISA (). The dashed line is the positive cutoff. NC: negative control. PC: positive control

tory with symptomatic individuals, COVID-19 was suspected, and the initial SARS-CoV-2 PCR test was indeed positive. Although multiple PCR tests performed afterward were negative, we could not prove that the initial result had been a false positive. Furthermore, as the patient continued to have a fever, it was difficult to arrive at a diagnosis and remove the patient from isolation.

Pleural effusion is an atypical symptom of COVID-19 and has been reported in a few cases of the disease. However, these cases of pleural effusion are largely pneumoniaassociated and do not occur in instances where the pneumonia image is unremarkable (4). There have been several reports on the overlap between COVID-19 and tuberculosis infection $(5,6)$. In one such report, 49 cases from 8 countries showed overlap between COVID-19 and tuberculosis. The median age of the 49 patients was 48 years old, 40 $(81.6 \%)$ were men, $48(98.0 \%)$ had pulmonary tuberculosis, and 3 had pleurisy. Typical symptoms were a fever, cough, and dyspnea. Bilateral frosted shadows were observed in 21 (47.7\%) cases (5). Although the course details of each case are unknown, there is a possibility of overlapping tuberculous pleurisy and COVID-19 without frosted shadows in the lung fields.

As of August 2021, the COVID-19 tests available in Japan include antigen tests (PCR (7), LAMP (8)), antibody tests (ELISA (3), immunochromatography (9)), and culture tests. PCR is considered to be extremely specific (10), with a review report showing the specificity to be over $99 \%$ (11). However, the sensitivity and specificity of the COVID-19 tests are not $100 \%$, and false positives may occur. In a multicenter survey conducted by The Infectious Diseases Society of Japan, 125 cases of suspected false positives were reported in 61 institutions (12). Although viral culture tests are considered to be highly sensitive, they are difficult to perform in actual clinical practice (13).

The COVID-19 pandemic is ongoing as of August 2021, and many hospitals are now testing all scheduled inpatients to prevent nosocomial COVID-19 infection. The large number of tests performed on patients with a low probability of prior infection is thought to increase the number of falsepositive cases. Likewise, because there are many conditions similar to COVID-19, the number of cases with an incorrect diagnosis of COVID-19 is increasing.

A false-positive result may lead to unnecessary additional tests and infection risk in patients admitted to COVID-19 wards. Furthermore, it results in psychological stress over the possibility of having COVID-19, leading to the unnecessary consumption of material resources due to prolonged isolation and a delayed diagnosis of the true disease (14).

Unfortunately, it remains difficult to scientifically prove false positives. As of August 2021, the testing system has expanded, and commercial antibody testing by companies is available. However, these antibody tests have not yet been established as diagnostic because they do not show an increase in $\operatorname{IgG}$ or $\operatorname{IgM}$ titers until approximately two weeks have passed (15). In addition, a high antibody titer is not directly related to the presence of an infection. Hence, an antibody test cannot always prove a false-positive result in PCR tests.

During the medical examination of the present case in May 2020, we were only able to perform PCR in actual clinical practice. We performed antigen PCR using nasal swabs and pleural fluids. Antibody testing with blood was performed to validate the results (Table 2). We considered the possibility that the antigen PCR assay at the time of admission of our patient had been a false-positive, as there was no increase in antibody titer; however, we were unable to completely rule out a SARS-CoV-2 infection.

Typical causes of false-positive PCR results are specimen contamination during collection or the pretreatment stage of the test (16). In the qualitative antigen test, the possibility of cross-reactivity with pathogens other than the new coronavirus (e.g. rhinovirus) has been pointed out, especially in small children (17). However, whether or not the same possibility exists with PCR is unclear. If false positives persist at the same facility, the possibility of specimen contamination should be checked first, and if contamination is suspected, the testing system should be reviewed. Even in the present case, we considered the possibility of contamination and made inquiries with the laboratory; however, we were unable to identify the actual cause.

In addition to false positives, false-negative results are also common in COVID-19 tests. False negatives create a variety of problems, including the risk of nosocomial cluster outbreaks and a lack of appropriate treatment when a patient is admitted to a general hospital bed. A meta-analysis study reported a summary estimate of the false-negative rate as $13 \%$ (95\% confidence interval: $9 \%-19 \%$ ); however, the reported values varied widely among studies, and the data were unclear (18). If a patient has a contact history with a COVID-19-positive individual or has symptoms of COVID19, the patient should be considered a false-negative. As 
with false positives, there is no way to reliably prove a false negative. If a false-negative result is suspected, additional tests should be performed, and other disease diagnoses should be considered.

False-positive results affect the criteria for removal of the patient from isolation and hospital discharge in clinical practice. As of May 2020, when our patient was being treated, the discharge criteria for COVID-19 infection in Japan were 2 negative PCR results after at least $24 \mathrm{~h}$ of no fever or upper respiratory tract symptoms. In June 2020, the discharge criteria were revised to "Patients can be discharged if 10 days have passed since the onset of the symptoms and 72 hours have passed since the symptoms were resolved". However, in our case, the patient continued to have febrile symptoms, and it was difficult to lift the isolation, even based on the current hospital discharge criteria. Some countries require PCR testing, while others do not, and no common discharge criteria have yet been (19). In Japan, the final decision on public health issues, such as lifting quarantine, is carried out with the cooperation of the public health center. For instance, because we concluded that the persistent fever in this case was due to tuberculous pleurisy, we discussed the criteria for discharge with the public health center and decided that the patient could be discharged after 10 days had passed since the onset of the illness with 2 negative PCR tests. If it is suspected that the PCR result is a false positive, as in our case, the usual COVID-19 discharge criteria should not be applied.

In conclusion, although SARS CoV-2 PCR is a highly sensitive and specific test, there is a possibility of falsepositive and false-negative results. As COVID-19 continues to spread, the number of false-positive cases is likely to increase. There is no clear way to prove a false-positive result, and the final diagnosis must be made by the clinician in a comprehensive manner. In addition, cooperation with the public health department is essential in dealing with public health issues, such as the lifting of isolation and discharge of patients with infectious diseases.

The authors state that they have no Conflict of Interest (COI).

\section{Acknowledgements}

We thank Drs. Mikako Ueno and Yoichi Teratake (Department of Intractable Diseases, National Center for Global Health and Medicine) for supplying the antigen proteins for ELISAs. This work was supported by the Japan Agency for Medical Research and Development [JP19fk0108163 and JP20fk0108160].

\section{References}

1. WHO Coronavirus Disease (COVID-19) Dashboard [Internet]. [cited $2020 \mathrm{Jul}$ 27]. Available from: https://covid19.who.int/.

2. Du Y, Tu L, Zhu P, et al. Clinical Features of 85 Fatal Cases of COVID-19 from Wuhan. A Retrospective Observational Study. Am J Respir Crit Care Med 201 (11): 1372-1379, 2020.

3. Satoshi K, Yusuke A, Akihiro M. Loss of Anti-SARS-CoV-2 Anti- bodies in Mild Covid-19. N Engl J Med 383 (17): 1694-1698, 2020.

4. S S, A A, S B, A G. Coronavirus Disease 2019 (COVID-19): A Systematic Review of Imaging Findings in 919 Patients. AJR Am J Roentgenol 215 (1): 87-93, 2020.

5. Tadolini M, Codecasa LR, García-García J-M, et al. Active tuberculosis, sequelae and COVID-19 co-infection: first cohort of 49 cases. Eur Respir J 56 (1): 2020.

6. $\mathrm{G} \mathrm{H}, \mathrm{J} \mathrm{W}, \mathrm{J}$ S, et al. COVID-19 in tuberculosis patients: A report of three cases. J Med Virol 92 (10): 1802-1806, 2020.

7. Manual for the Detection of Pathogen 2019-nCoV Ver.2.6 [Internet]. [cited $2020 \mathrm{Jul}$ 27]. Available from: https://www.niid.go.jp/nii d/images/epi/corona/2019-nCoVmanual20200217-en.pdf.

8. Kitagawa Y, Orihara Y, Kawamura R, et al. Evaluation of rapid diagnosis of novel coronavirus disease (COVID-19) using loopmediated isothermal amplification. J Clin Virol 129: 2020.

9. National Institute of Infectious Diseases. Detection of anti-SARSCoV-2 antibodies in COVID-19 patients using a lateral flow rapid test. Published 2020 [Internet]. [cited 2020 Jul 27]. Available from: https://www.niid.go.jp/niid/ja/diseases/ka/corona-virus/2019ncov/9520-covid19-16.html.

10. Tsukadaira A. The specificity of Japanese PCR assays for SARSCoV-2 exceeds 99.7\%. medRxiv Published online May 20, 2020: 2020.05.15.20103515.

11. J D, JJ D, A A, et al. Rapid, point-of-care antigen and molecularbased tests for diagnosis of SARS-CoV-2 infection. Cochrane database Syst Rev 8 (8): 2020.

12. Results of a Questionnaire on False Positives in COVID-19 Simple Antigen Assay Test [Internet]. [cited 2021 Sep 2]. Available from: https://www.kansensho.or.jp/uploads/files/topics/2019ncov/co vid19_survey_201027.pdf.

13. Laboratory biosafety guidance related to coronavirus disease (COVID-19) [Internet]. [cited 2020 Jul 25]. Available from: http s://www.who.int/publications/i/item/laboratory-biosafety-guidance-r elated-to-coronavirus-disease-2019-(covid-19).

14. E S, V N, F D. False-positive COVID-19 results: hidden problems and costs. Lancet Respir Med 8 (12): 1167-1168, 2020.

15. Takaku Yotaro, Kurashima Kazuyoshi, Ishiguro Takashi, et al. Antibody Response as Assessed by A Rapid Immunochromatographic Assay in Patients with COVID-19 Pneumonia Caused by the Novel Coronavirus, SARS-CoV-2. Kansenshogaku Zasshi 94 (4): 495-499, 2020.

16. Cohen AN, Kessel B. False positives in reverse transcription PCR testing for SARS-CoV-2. medRxiv Published online May 1, 2020: 2020.04.26.20080911.

17. Otake S, Miyamoto S, Mori A, Iwamoto $T$, Kasai M. Falsepositive results in SARS-CoV-2 antigen test with rhinovirus-A infection. Pediatr Int 63 (9): 1135-1137, 2021.

18. I A-R, D B-G, D S-R, et al. False-negative results of initial RTPCR assays for COVID-19: A systematic review. PLoS One 15 (12): 2020.

19. Centre for Disease Prevention E. Guidance for Discharge and Ending Isolation in the Context of Widespread Community Transmission of COVID-19-First Update Scope of This Document; 2020 [Internet]. [cited $2020 \mathrm{Jul}$ 26]. Available from: https://www.ecdc.eu ropa.eu/sites/default/files/documents/covid-19-guidance-discharge-a nd-ending-isolation-firstupdate.pdf.

The Internal Medicine is an Open Access journal distributed under the Creative Commons Attribution-NonCommercial-NoDerivatives 4.0 International License. To view the details of this license, please visit (https://creativecommons.org/licenses/ by-nc-nd/4.0/). 
(C) The Japanese Society of Internal Medicine Intern Med Advance Publication 